\title{
Assessment of lymph nodes and prostate status using early dynamic curves with ${ }^{18} \mathrm{~F}$-choline PET/CT in prostate cancer
}

\author{
Cédric Mathieu ${ }^{1,2 *}$, Ludovic Ferrer ${ }^{3,4}$, Thomas Carlier ${ }^{2,3}$, Mathilde Colombié ${ }^{1}$, \\ Daniela Rusu ${ }^{1}$, Françoise Kraeber-Bodéré ${ }^{1,2,3}$, Loic Campion ${ }^{5}$ and Caroline Rousseau ${ }^{1,3}$
}

${ }^{1}$ Department of Nuclear Medicine, ICO Cancer Center, Saint Herblain, France, ${ }^{2}$ Department of Nuclear Medicine, University Hospital, Nantes, France, ${ }^{3}$ Centre Régional de Recherche en Cancérologie Nantes/Angers, U892, CNRS UMR 6299, INSERM, Nantes, France, ${ }^{4}$ Department of Medical Physics, ICO Cancer Center, Saint Herblain, France, ${ }^{5}$ Department of Statistics, ICO Cancer Center, Saint Herblain, France

OPEN ACCESS

Edited by:

Thierry M. L. B. Vander Borght, CHU Dinant Godinne, Belgium

Reviewed by:

Maria Mathew D'Souza, Institute of Nuclear Medicine and Allied Sciences, India Baljinder Singh,

Post Graduate Institute of Medical Education and Research, India

${ }^{*}$ Correspondence: Cédric Mathieu,

Department of Nuclear Medicine, ICO Cancer Center, Boulevard Jacques Monod, Saint Herblain 44805, France cedric.mathieu@chu-nantes.fr

Specialty section: This article was submitted to Nuclear Medicine, a section of the journal Frontiers in Medicine

Received: 29 April 2015 Accepted: 27 August 2015 Published: 09 September 2015

Citation:

Mathieu C, Ferrer L, Carlier T, Colombié M, Rusu D,

Kraeber-Bodéré F, Campion $L$ and Rousseau C (2015) Assessment of lymph nodes and prostate status using early dynamic curves with

${ }^{18} \mathrm{~F}$-choline PET/CT in prostate cancer.

Front. Med. 2:67.

doi: 10.3389/fmed.2015.00067
Introduction: Dynamic image acquisition with ${ }^{18} \mathrm{~F}$-Choline [fluorocholine $(\mathrm{FCH})$ ] PET/CT in prostate cancer is mostly used to overcome the bladder repletion, which could obstruct the loco-regional analysis. The aim of our study was to analyze early dynamic $\mathrm{FCH}$ acquisitions to define pelvic lymph node or prostate pathological status.

Material and methods: Retrospective analysis was performed on 39 patients for initial staging $(n=18)$, or after initial treatment $(n=21)$. Patients underwent 10-min dynamic acquisitions centered on the pelvis, after injection of $3-4 \mathrm{MBq} / \mathrm{kg}$ of $\mathrm{FCH}$. Whole-body images were acquired about $1 \mathrm{~h}$ after injection using a PET/CT GE Discovery LS (GELS) or Siemens Biograph mCT (mCT). Maximum and mean SUV according to time were measured on nodal and prostatic lesions. SUV mean was corrected for partial volume effect (PVEC) with suitable recovery coefficients. The status of each lesion was based on histological results or patient follow-up (>6 months). A Mann-Whitney test and ANOVA were used to compare mean and receiver operating characteristic $(\mathrm{ROC})$ curve analysis.

Results: The median PSA was $8.46 \mathrm{ng} / \mathrm{mL}$ and the median Gleason score was $3+4$. Ninety-two lesions (43 lymph nodes and 49 prostate lesions) were analyzed, including 63 malignant lesions. In early dynamic acquisitions, the maximum and mean SUV were significantly higher, respectively, on $\mathrm{MCT}$ and GE-LS, in malignant versus benign lesions $(p<0.001, p<0.001)$. Mean SUV without PVEC, allowed better discrimination of benign from malignant lesions, in comparison with maximum and mean SUV (with PVEC), for both early and late acquisitions. For patients acquired on $\mathrm{mCT}$, area under the ROC curve showed a trend to better sensitivity and specificity for early acquisitions, compared with late acquisitions (SUVmax AUC 0.92 versus 0.85, respectively).

Conclusion: Assessment of lymph nodes and prostate pathological status with early dynamic imaging using PET/CT FCH allowed prostate cancer detection in situations where proof of malignancy is difficult to obtain.

Keywords: ${ }^{18} \mathrm{~F}$-choline, prostate cancer, dynamic curves, early acquisition, pathological status 


\section{Introduction}

Prostate cancer is the most common cancer in men over 50 years, and the third highest cause of death by cancer. Initial staging is reserved for tumors with intermediate or high risk according to the D'Amico classification (1). Recurrence of prostate cancer after treatment is common and occurs in $20-50 \%$ of cases at 10 years after radical prostatectomy, and in $30-40 \%$ after radiotherapy $(2,3)$. Management of biological relapse after curative treatment is a real diagnostic and therapeutic challenge. Hormone therapy is currently the standard palliative treatment and resistance inevitably occurs after 2-5 years (4).

In patients with intermediate or high risk, local extension, nodal, or bone metastasis, have major prognostic and therapeutic implications (4). Bone scans are recommended for patients at intermediate or high risk of progression. MRI has a major role for local extension, or location of prostate cancer after negative biopsies (5-7). Abdominal and pelvic CT can be used in order to highlight nodal metastases (8). However, imaging is supplanted by extent lymphadenectomy, which is recommended before prostatectomy or radiotherapy for intermediate and high risk prostate cancer to precisely evaluate the nodal status (9). The sentinel lymph node technique is being evaluated to guide surgeons to ensure optimal quality of dissection (10).

In prostate cancer, fluorocholine $(\mathrm{FCH}) \mathrm{PET} / \mathrm{CT}$ is indicated as an alternative to FDG PET/CT relevant to mitotic and choline kinase activities $(11,12)$. FCH is a highly sensitive and specific radiopharmaceutical for the initial staging of prostate cancer (intermediate or high risk tumors), or suspicion of recurrence (13-16). In most cases, PET/CT is performed in two phases (17). The first is a kinetic step centered on the pelvis to achieve the regional analysis before bladder repletion. The second consists of whole-body image acquisition $1 \mathrm{~h}$ post-injection.

The aim of our study was to evaluate the potential benefit of early kinetic FCH PET/CT for discriminating malignant from benign lymph node or prostate lesions, whose status has been proven by histological analysis or patient follow-up.

\section{Materials and Methods}

\section{Population}

Retrospective analyses were performed on patients with histologically proven prostatic adenocarcinoma and explored for initial staging or biochemical recurrence. Recurrence was defined as two consecutive PSA values of $0.2 \mathrm{ng} / \mathrm{mL}$ and above after radical prostatectomy, or three consecutive increasing PSA values above the previous PSA nadir measured at 3-month interval after radiotherapy. Patients had at least one focal FCH uptake in pelvic lymph nodes or in the prostate. All patients had an initially negative or equivocal conventional imaging, including bone scan, CT, and/or MRI. For each patient, we collected age, serum PSA, date of initial diagnosis, Gleason score, topography of prostate cancer, initial treatment, and time to recurrence. We obtained informed consent from all patients allowing the use of their clinical data for research purposes under a protocol approved in our institution.

\section{Acquisition and Interpretation of PEC/CT}

After $6 \mathrm{~h}$ of fasting, 3-4 MBq/kg of FCH were injected when starting PET/CT acquisition. Acquisition was realized in two phases. First a 10-min kinetic acquisition in list mode centered on the pelvis was acquired, followed by whole-body image acquisition at $60 \mathrm{~min}$. Examinations were performed on two different PET/CT instruments, a GE Discovery LS (GE-LS) (GE Medical System, Waukesha WI, USA) and a Siemens Biograph mCT 40 (mCT) (Siemens, Erlanger, Germany). Images were reconstructed by iterative OSEM reconstruction with two iterations and 28 subsets associated with a 2D Gaussian filter (FWHM $5.45 \mathrm{~mm}$ ) for GELS and OP-OSEM-PSF TOF with 3 iterations and 21 subsets associated with a 3D Gaussian filter ( $2 \mathrm{~mm}$ FWHM) for mCT.

Maximum and mean standard uptake values $\left(\mathrm{SUV}_{\max }\right.$ and $\left.\mathrm{SUV}_{\text {mean }}\right)$ were measured on late images in every prostatic or pelvic nodal lesion. Dynamic curves were built for each lesion, measuring the $\mathrm{SUV}_{\max }$ and $\mathrm{SUV}_{\text {mean }}$ every minute in the first $10 \mathrm{~min}$. To delineate the tumor contours, we used a systemspecific contrast-oriented algorithm proposed by Nestle (18), using the following formula:

$$
\mathrm{SUV}_{\text {threshold }}=\left(k \times \mathrm{SUV}_{\text {max }}\right)+\mathrm{SUV}_{\text {background }}
$$

The $\mathrm{SUV}_{\text {background }}$ was defined as the $\mathrm{SUV}_{\text {mean }}$ in the gluteus maximus, and $k$ was a system-specific constant determined by phantom acquisitions for each PET/CT camera. A correction of partial volume effect (PVEC) on $\mathrm{SUV}_{\text {mean }}$ was performed with suitable recovery coefficients (19).

\section{Gold Standard and Statistical Analyses}

Lymph node or prostate lesions preferentially confirmed by histology were considered (prostatectomy results, lymph node dissection, or prostate biopsies). In other cases, concordant imaging (CT or MRI) or PSA decrease after targeted radiotherapy was also used to confirm lesion status. FCH uptake in the inguinal region was interpreted as reactive inflammatory lymph node as described in the literature (20). For patients with prostatic histological proof (biopsy or resection), the agreement between PET images and histology was evaluated by comparison with the detailed histological report. Statistical analysis consisted of a comparison of repeated averages between benign and malignant lesions using an ANOVA for early acquisitions. For late acquisitions, the SUV of benign and malignant lesions were compared by comparing averages (non-parametric Mann-Whitney test). SUV between the end of the early acquisition ( $10 \mathrm{~min}$ ) and the late acquisition (60 min) was compared using a paired $t$-test. Early and late acquisitions were compared using a receiver operating characteristic (ROC) curve, only for patients acquired on $\mathrm{mCT}(n=71)$. The tests were performed bilaterally with a limit of $5 \%$ significance $(p \leq 0.05)$. The software used was SPSS 18 and Stata 13.1.

\section{Results}

\section{Patients and Lesions}

We identified 39 patients from September 2008 to January 2014 with FCH uptake in 49 prostatic and 43 nodal lesions. Median 
age was 73 years and median PSA at PET time was $8.46 \mathrm{ng} / \mathrm{mL}$ (2.02-172.6). Among the 21 patients with biochemical recurrence, initial treatment was a prostatectomy for $38 \%(8 / 21)$ of them, and the median time to recurrence was 54.8 months (12-195). The median PSA doubling time was 8.2 months (1.7-24.7). Four patients were treated with hormone therapy at the time of PET (10.2\%). Patient characteristics are presented in Table 1.

Within the 39 patients explored, 19 patients (49\%) had isolated prostatic lesions, $8(20 \%)$ isolated nodal lesions, and $12(31 \%)$ had both. Histological results were available for $96.7 \%(30 / 31)$ of patients with prostatic lesions and for $40 \%(8 / 20)$ of patients with nodal lesions (lymph node dissection). For one patient with histologically unconfirmed prostatic lesions, PSA decrease after targeted radiotherapy was considered as a proof of disease. For the 12 patients with histologically unproven lymph node lesions, malignancy was assessed by a concordant imaging between CT and MRI in five cases (25\%), PSA decrease after targeted radiotherapy in three cases $(15 \%)$ or inguinal lymph node location in four cases $(20 \%)$. Lesion characteristics are presented in Table 2.

TABLE 1 | Description of patient characteristics $(n=39)$.

\begin{tabular}{lcc}
\hline & $\begin{array}{c}\text { Initial staging } \boldsymbol{n}=\mathbf{1 8} \\
\text { (Min-Max) }\end{array}$ & $\begin{array}{c}\text { Recurrence } \boldsymbol{n}=\mathbf{2 1} \\
\text { (Min-Max) }\end{array}$ \\
\hline Median age (years) & $70.5(51-78)$ & $74(55-84)$ \\
Median PSA at PET (ng/mL) & $11.5(3.5-172.6)$ & $5.23(2.02-14.95)$ \\
Gleason score & & 5 \\
6 & 3 & 13 \\
7 & 9 & 2 \\
8-9 & 6 & 1 \\
Unknown & 0 & 3 \\
Initial stage & & 10 \\
T1 & 7 & 5 \\
T2 & 6 & 3 \\
T3 & 3 & 2 \\
Unknown & 2 & 5 \\
Previous treatment & & 1 \\
S & NA & 10 \\
S+ R & & 1 \\
S+HT & & 2 \\
R & & \\
R+HT & & \\
HT & & \\
& & \\
\hline
\end{tabular}

$S$, surgery; $S+R$, Surgery + Radiotherapy; $R+H T$, Radiotherapy + Hormone Therapy; $H T$, hormone therapy; NA, not applicable.
Within the 49 prostatic lesions, 38 were malignant (77.6\%), and $28(57.1 \%)$ were identified in patients referred for initial staging. Forty-three nodal lesions were explored, 25 were malignant (58.1\%) and 18 (41.8\%) were identified in patients referred for initial staging. The location of the 25 malignant lymph nodes was as follows: common iliac for 5 (20\%), external iliac for $14(56 \%)$, internal iliac for $2(8 \%)$, obturator fossa for $1(4 \%)$, and pre-sacral for $3(12 \%)$. Benign lymph nodes were found in the common iliac area for one $(6 \%)$, external iliac for five $(28 \%)$, and inguinal node for $12(66 \%)$.

\section{Early Kinetic of Lymph Node and Prostate Lesions}

The mean $\mathrm{SUV}_{\max }$ on the early dynamic acquisitions was significantly higher for malignant versus benign lesions, respectively, on mCT $(n=71)$ and on GE-LS $(n=21)(p<0.001$ and $p<0.001)$. Malignant lesions showed intense FCH uptake, with a maximum level of $\mathrm{SUV}_{\max }$ almost reached at the second minute post-injection, followed by a plateau. Benign lesions showed a less intense uptake. The mean $\mathrm{SUV}_{\max }$ in the plateau was, respectively, on mCT and on GE-LS about 12 and 8 for malignant lesions and 5 and 3 for benign lesions (Figures 1 and 2). The results were confirmed with $\mathrm{SUV}_{\text {mean }}$ with significantly higher $\mathrm{FCH}$ uptake in malignant lesions (with and without PVEC).

\section{Analysis of 60 min Post-Injection Images and Comparison to the Early Kinetic Results}

On mCT $(n=71)$, the mean $\mathrm{SUV}_{\max }$ in the late acquisition was significantly higher in malignant lesions versus benign lesions, 11.1 versus $3.8(p<0.001)$, and also on LS-GE PET $(n=21) 8$ versus $2.7(p<0.001)$. There was a significant decrease of the average $S U V_{m a x}$ between the end of the early acquisition and late acquisition of $13 \%$ for malignant lesions (12.8 versus 11.1, $p<0.001$ ), and $19 \%$ for benign lesions (4.7 versus 3.8, $p=0.02$ ).

On mCT $(n=71)$, the optimal SUV threshold that maximized both sensitivity and specificity in early acquisitions was obtained

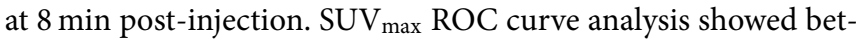
ter area under the curve (AUC) for the early acquisition at $8 \mathrm{~min}$ versus late acquisition (Figure 3) (0.92 versus 0.85 , respectively). In the early acquisitions, $\mathrm{SUV}_{\text {mean }}$ displayed the best AUC in comparison with $\mathrm{SUV}_{\max }$ and $\mathrm{SUV}_{\text {mean }}$ with PVEC (0.97 versus 0.92 and 0.91 , respectively). The same results were found for late acquisitions with a superiority of $\mathrm{SUV}_{\text {mean }}$ without PVEC. Optimal threshold and data for $\mathrm{SUV}_{\text {mean }}$ with and without PVEC are summarized in Table 3.

TABLE 2 | Description of lesion characteristics $(n=92)$ in the 39 patients.

\begin{tabular}{|c|c|c|c|c|}
\hline & \multicolumn{2}{|c|}{ Prostatic lesions number (\%) } & \multicolumn{2}{|c|}{ Nodal lesions number (\%) } \\
\hline & Per patient analysis & Per lesion analysis & Per patient analysis & Per lesion analysis \\
\hline Number & 31 & 49 & 20 & 43 \\
\hline Histological proof & $30(97 \%)$ & 48 (98\%) & $8(40 \%)$ & 14 (33\%) \\
\hline Concordant imaging & 0 (0\%) & $0(0 \%)$ & $5(25 \%)$ & $14(33 \%)$ \\
\hline PSA decrease after RTE & $1(3 \%)$ & $1(2 \%)$ & $3(15 \%)$ & $7(16 \%)$ \\
\hline
\end{tabular}

$R T E$, radiotherapy. 

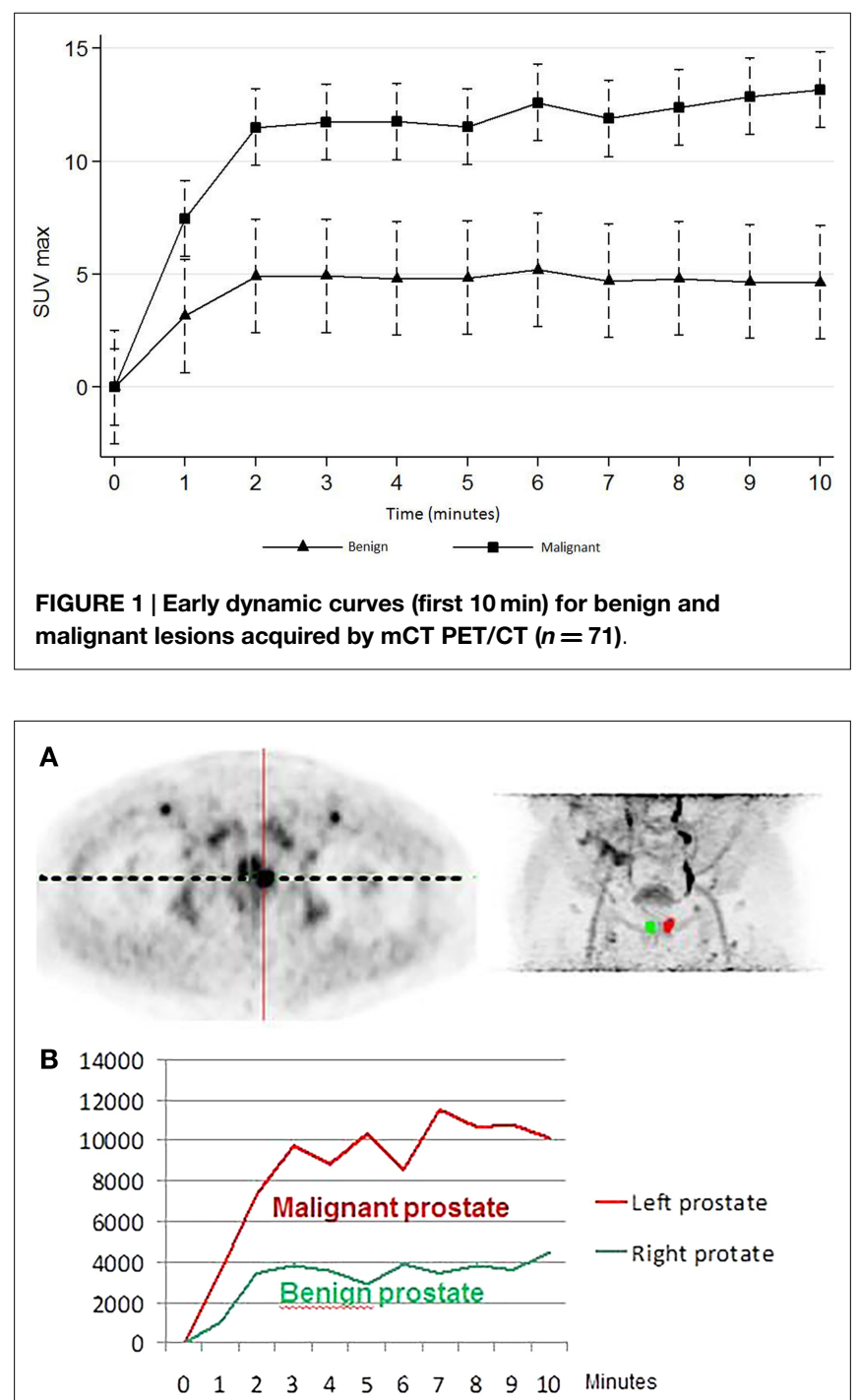

FIGURE 2 | (A) Axial early FCH PET/CT acquisition (mCT PET/CT instrument), (B) maximum intensity projection (MIP) in the same patient. Patient evaluated before initial surgery with a prostatic adenocarcinoma, Gleason $3+4$, PSA at $10 \mathrm{ng} / \mathrm{mL}$. Histological analysis showed a left prostatic adenocarcinoma (red curve) while the right lobe was free of disease (green curve): statistically significant difference of tracer uptake between the two lobes was obtained.

\section{Discussion}

We conducted a retrospective study with early dynamic acquisitions of FCH PET/CT in patients explored for initial staging or suspicion of recurrence of prostate cancer. To the best of our knowledge, this is the first study to assess the kinetic uptake of the radiopharmaceutical with 1 min step images to discriminate benign from malignant lesions, and explore the potential benefit that could be provided by PVEC correction for $\mathrm{SUV}_{\text {mean }}$.

There are few published data on the contribution of dynamic acquisitions, and most involve small cohorts of patients. While it is known that an initial dynamic acquisition is useful in differentiating prostatic lesions from the urinary tract (21), the optimal image acquisition time remains uncertain. For prostatic lesions, Kwee et al. (17) conducted a prospective study in 26 patients with an early whole-body imaging at 7 min post-injection associated with a step on the pelvis $1 \mathrm{~h}$ post-injection. They showed that benign lesions could be quickly differentiated from malignant lesions with higher uptake and an increased SUV $\mathrm{max}_{\max }$ by $14 \%$ over time. Because they built a curve with two independent measurements of SUV, they could not precisely define the time to reach maximum activity. Steiner et al. (22) in a retrospective study of 15 lesions in 11 patients achieved a FCH PET/CT in 3 phases (dynamic $10 \mathrm{~min}$, immediately followed by a whole body and a late acquisition on pelvis at $1 \mathrm{~h}$ post-injection). They found that benign lesions could be quickly differentiated from malignant lesions, which show higher uptake and an increasing $\mathrm{SUV}_{\max }$ until the 14th minute. In our study, the dynamic acquisition in list mode allowed us to determine a maximum activity reached in about the third minute post-injection, followed by an initial plateau and a discrete reduction of SUV, especially for benign lesions.

Dynamic acquisitions with choline labeled with ${ }^{18}$ Fluorine are more appropriate than with ${ }^{11}$ Carbon, because of the short halflife of ${ }^{11}$ Carbon $(20 \mathrm{~min})$. However, a prospective study using ${ }^{11} \mathrm{C}$-Choline in 56 patients performed with two successive wholebody acquisitions at about $3-5$ and 20 min post-injection, demonstrated dual phase acquisition was useful in distinguishing benign from malignant lesions (23). Nevertheless, they used a different radiopharmaceutical and they did not acquire dynamic, therefore making comparisons to our study difficult.

The behavioral differences between malignant and benign lesions, whether for prostatic or nodal lesions, encourage the use of ROC curves to define optimal thresholds to discriminate the lesions. For prostatic lesions, Kwee et al. (17) found an AUC of 0.81 at $7 \mathrm{~min}$ and 0.92 for late acquisition at $1 \mathrm{~h}$, without defining a threshold. Oprea-Lager et al. (20) only examined nodal lesions, with an AUC of 0.93 at $30 \mathrm{~min}$ for the $\mathrm{SUV}_{\max }$ and 0.92 for the SUV $V_{\text {mean. }}$ They determined thresholds for lymph nodes at 2.32 and 1.66 on late acquisitions for $\mathrm{SUV}_{\max }$ and $\mathrm{SUV}_{\text {mean }}$, respectively. In our study, we found similar AUC using $S \mathrm{SV}_{\max }$ for early and late acquisitions ( 0.92 and 0.85 , respectively). Our

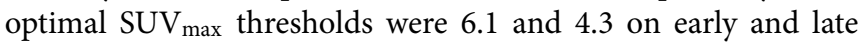
acquisitions, respectively, which allowed a sensitivity of 89.8 and $77.5 \%$, and a specificity of 90.9 and $77.3 \%$. We observed superior performance for $\mathrm{SUV}_{\text {mean }}$ than $\mathrm{SUV}_{\text {max }}$, for both early and late acquisitions. We also observed a better AUC for the early acquisition at $8 \mathrm{~min}$ versus late acquisition. Few studies explored the use of $\mathrm{SUV}_{\text {mean }}$, especially for response to therapy studies with a high impact of PVE correction. Nevertheless, a precise and robust delineation of tumor functional volume as used in our study, this index seems more relevant than $S U V_{\max } . S V_{\max }$ provides only very limited information relating to radiotracer accumulation, and no information on the associated tumor uptake distribution or the overall tumor functional volume (24). A meta-analysis showed better repeatability performance of $S_{U V} V_{\text {mean }}$ versus $S_{U V} V_{\max }$ and could explain the differences observed (25).

We acknowledge that our study has some limitations. First, our acquisitions used two different generation PET/CT 
A

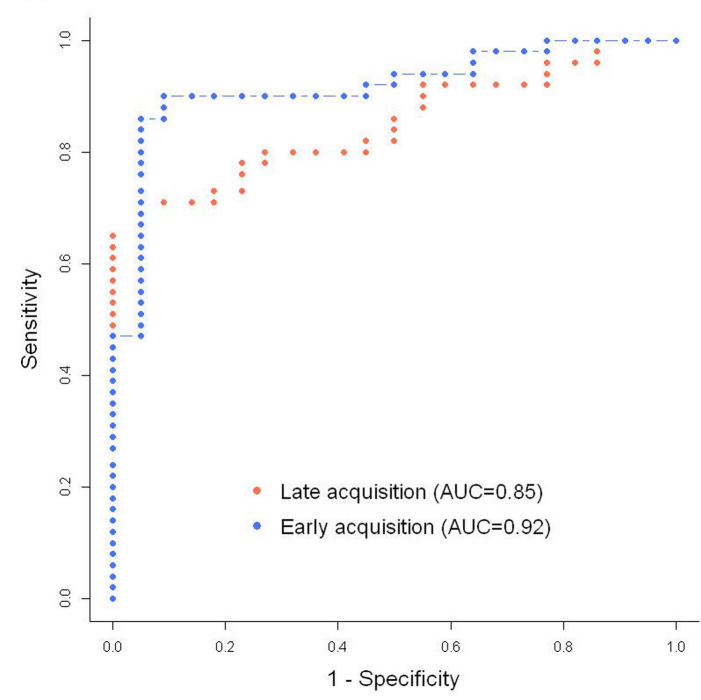

B

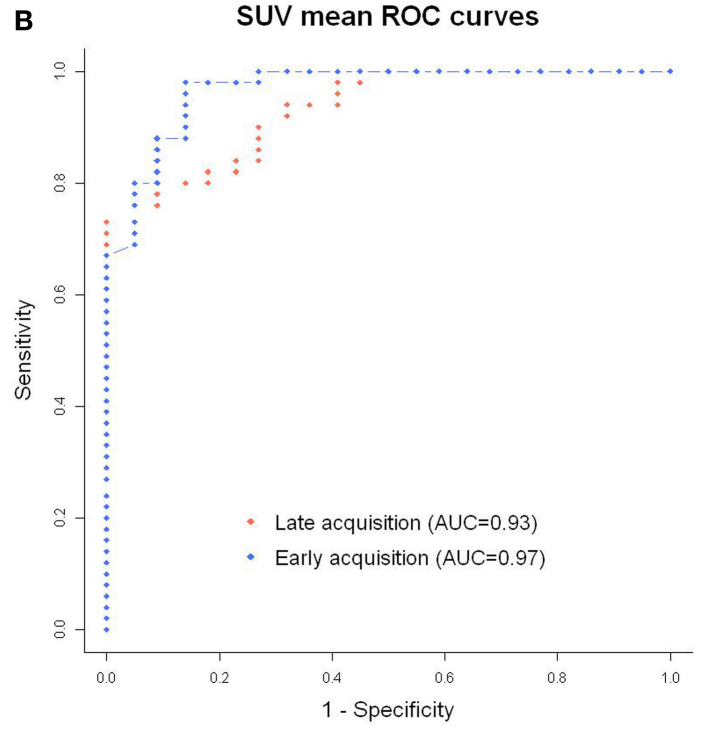

FIGURE 3 | Receiver operating characteristic curve for distinguishing benign from malignant lesions using SUV max $_{(A)}$ and SUV $_{\text {mean }}[(B)$, without partial volume effect correction] for patients acquired on $\mathrm{mCT}(n=71)$.

TABLE 3 | Optimal threshold of SUV for distinguishing benign from malignant lesions using SUV $_{\text {max }}$ and $S U V_{\text {mean }}$ for patients acquired on mCT ( $n=71$ )

\begin{tabular}{|c|c|c|c|c|c|c|}
\hline & Acquisition & Optimal threshold & AUC & Sensitivity (\%) & Specificity (\%) & Accuracy (\%) \\
\hline \multirow[t]{2}{*}{ SUV $\max$} & Early & 6.1 & 0.92 & 89.8 & 90.9 & 90.1 \\
\hline & Late & 4.3 & 0.85 & 77.5 & 77.3 & 77.5 \\
\hline \multirow[t]{2}{*}{ SUV $V_{\text {mean }}$} & Early & 2.5 & 0.97 & 98 & 86.4 & 94.4 \\
\hline & Late & 2.4 & 0.93 & 89.8 & 72.7 & 84.5 \\
\hline \multirow[t]{2}{*}{$S_{\text {SUV }}$ mean $(P V E C)$} & Early & 5.1 & 0.91 & 87.8 & 81.8 & 85.9 \\
\hline & Late & 5.0 & 0.87 & 83.7 & 72.7 & 80.3 \\
\hline
\end{tabular}

instruments. This resulted in significant differences in detection sensitivity, making it impossible to compare patients acquired on the two different PET/CT systems. Second, as our study was based on patients where the malignant or benign status of lesions was proven, the enrollment was limited but equally important compared to other literature studies. For prostate lesions, we obtained a high rate of histological proof; $96.7 \%$ of patients had prostate lesions proven by biopsy or prostatectomy. For lymph node involvement, histological results were obtained for only $40 \%$ of patients, which was partially due to the difficulty in proposing systematic lymph node dissection based on PET/CT results. On the other hand, it was difficult to intraoperatively identify lymph node lesions detected by PET/CT because most of the time FCH positive lymph nodes did not correspond to morphologically increased node volume. Oprea-Lager et al. reported the same difficulties in obtaining proof for suspected malignant lymph nodes (20).

\section{References}

1. Heidenreich A, Bastian PJ, Bellmunt J, Bolla M, Joniau S, van der Kwast T, et al. EAU guidelines on prostate cancer. Part 1: screening, diagnosis, and local treatment with curative intent-update 2013. Eur Urol (2014) 65:124-37. doi:10.1016/j.eururo.2013.09.046

\section{Conclusion}

Assessment of lymph nodes and prostate pathological status with early PET/CT FCH dynamic curves was successful in prostate cancer where proof of malignancy is difficult to obtain. In addition to avoiding bladder repletion, dynamic early acquisitions demonstrated intense and stable FCH uptake from the first minutes postinjection, and discriminated benign from malignant lesions either in prostate or in lymph nodes. $\mathrm{SUV}_{\text {mean }}$ without correction of PVEC and early acquisitions may perform better than maximum $\mathrm{SUV}_{\max }$ and late acquisitions, but larger studies are warranted.

\section{Acknowledgments}

This work has been supported in part by grants from the French National Agency for Research called "Investissements d'Avenir" Labex IRON no. ANR-11-LABX-0018-01.

2. Aus G, Abbou CC, Bolla M, Heidenreich A, Schmid H-P, van Poppel H, et al EAU guidelines on prostate cancer. Eur Urol (2005) 48:546-51. doi:10.1016/j. eururo.2005.06.001

3. Seltzer MA, Barbaric Z, Belldegrun A, Naitoh J, Dorey F, Phelps ME, et al. Comparison of helical computerized tomography, positron emission tomography and monoclonal antibody scans for evaluation of lymph node metastases in 
patients with prostate specific antigen relapse after treatment for localized prostate cancer. J Urol (1999) 162:1322-8. doi:10.1097/00005392-19991000000021

4. Heidenreich A, Bastian PJ, Bellmunt J, Bolla M, Joniau S, van der Kwast T, et al. EAU guidelines on prostate cancer. Part II: treatment of advanced, relapsing, and castration-resistant prostate cancer. Eur Urol (2014) 65:467-79. doi:10. 1016/j.eururo.2013.11.002

5. Bjurlin MA, Meng X, Le Nobin J, Wysock JS, Lepor H, Rosenkrantz AB, et al. Optimization of prostate biopsy: the role of MRI targeted biopsy in detection, localization, and risk assessment. J Urol (2014) 192(3):648-58. doi:10.1016/j. juro.2014.03.117

6. Murphy G, Haider M, Ghai S, Sreeharsha B. The expanding role of MRI in prostate cancer. AJR Am J Roentgenol (2013) 201:1229-38. doi:10.2214/AJR.12. 10178

7. Park JJ, Kim CK, Park SY, Park BK, Lee HM, Cho SW. Prostate cancer: role of pretreatment multiparametric 3-T MRI in predicting biochemical recurrence after radical prostatectomy. AJR Am J Roentgenol (2014) 202:W459-65. doi:10. 2214/AJR.13.11381

8. Hövels AM, Heesakkers RAM, Adang EM, Jager GJ, Strum S, Hoogeveen YL, et al. The diagnostic accuracy of CT and MRI in the staging of pelvic lymph nodes in patients with prostate cancer: a meta-analysis. Clin Radiol (2008) 63:387-95. doi:10.1016/j.crad.2007.05.022

9. Bastide C, Beuzeboc P, Cormier L, Fromont G, Hennequin C, Mongiat-Artus P, et al. Recommandations en onco-urologie 2013 du CCAFU: cancer de la prostate. Prog Urol (2013) 23:S67-101. doi:10.1016/S1166-7087(13)70048-4

10. Rousseau C, Rousseau T, Campion L, Lacoste J, Aillet G, Potiron E, et al. Laparoscopic sentinel lymph node versus hyperextensive pelvic dissection for staging clinically localized prostate carcinoma: a prospective study of 200 patients. J Nucl Med (2014) 55:753-8. doi:10.2967/jnumed.113.129023

11. Jadvar H. Prostate cancer: PET with $18 \mathrm{~F}-\mathrm{FDG}, 18 \mathrm{~F}$ - or $11 \mathrm{C}$-acetate, and $18 \mathrm{~F}$ - or 11C-choline. J Nucl Med (2011) 52:81-9. doi:10.2967/jnumed.110.077941

12. DeGrado TR, Baldwin SW, Wang S, Orr MD, Liao RP, Friedman HS, et al. Synthesis and evaluation of (18)F-labeled choline analogs as oncologic PET tracers. J Nucl Med (2001) 42:1805-14.

13. Evangelista L, Zattoni F, Guttilla A, Saladini G, Zattoni F, Colletti PM, et al. Choline PET or PET/CT and biochemical relapse of prostate cancer: a systematic review and meta-analysis. Clin Nucl Med (2013) 38:305-14. doi:10.1097/ RLU.0b013e3182867f3c

14. Beheshti M, Imamovic L, Broinger G, Vali R, Waldenberger P, Stoiber F, et al. $18 \mathrm{~F}$ choline $\mathrm{PET} / \mathrm{CT}$ in the preoperative staging of prostate cancer in patients with intermediate or high risk of extracapsular disease: a prospective study of 130 patients. Radiology (2010) 254:925-33. doi:10.1148/radiol.09090413

15. Krause BJ, Souvatzoglou M, Treiber U. Imaging of prostate cancer with PET/CT and radioactively labeled choline derivates. Urol Oncol (2013) 31:427-35. doi: 10.1016/j.urolonc.2010.08.008
16. Picchio M, Briganti A, Fanti S, Heidenreich A, Krause BJ, Messa C, et al. The role of choline positron emission tomography/computed tomography in the management of patients with prostate-specific antigen progression after radical treatment of prostate cancer. Eur Urol (2011) 59:51-60. doi:10.1016/j.eururo. 2010.09.004

17. Kwee SA, Wei H, Sesterhenn I, Yun D, Coel MN. Localization of primary prostate cancer with dual-phase 18F-fluorocholine PET. J Nucl Med (2006) 47:262-9.

18. Nestle U, Kremp S, Schaefer-Schuler A, Sebastian-Welsch C, Hellwig D, Rübe C, et al. Comparison of different methods for delineation of 18F-FDG PETpositive tissue for target volume definition in radiotherapy of patients with nonsmall cell lung cancer. J Nucl Med (2005) 46:1342-8.

19. Gallivanone F, Canevari C, Gianolli L, Salvatore C, Della Rosa PA, Gilardi MC, et al. A partial volume effect correction tailored for 18F-FDG-PET oncological studies. Biomed Res Int (2013) 2013:780458. doi:10.1155/2013/780458

20. Oprea-Lager DE, Vincent AD, van Moorselaar RJ, Gerritsen WR, Van den Eertwegh AJ, Eriksson J, et al. Dual-phase PET-CT to differentiate (18F) fluoromethylcholine uptake in reactive and malignant lymph nodes in patients with prostate cancer. PLoS One (2012) 7:e48430. doi:10.1371/journal.pone.0048430

21. Mohsen Beheshti WL. PET imaging of prostate cancer using radiolabeled choline. PET Clin (2009) 4:173-84. doi:10.1016/j.cpet.2009.06.003

22. Steiner C, Vees H, Zaidi H, Wissmeyer M, Berrebi O, Kossovsky MP, et al. Three-phase 18F-fluorocholine PET/CT in the evaluation of prostate cancer recurrence. Nuklearmedizin (2009) 48(1):1-9.

23. Garcia JR, Cuberas G, Riera E, Soler M, Moragas M, Lomeña F. Dual-phase 11C-choline PET/computed tomography in the early evaluation of prostate cancer recurrence. Nucl Med Commun (2015) 36:8-15. doi:10.1097/MNM. 0000000000000207

24. Visvikis D, Hatt M, Tixier F, Cheze Le Rest C. The age of reason for FDG PET image-derived indices. Eur J Nucl Med Mol Imaging (2012) 39:1670-2. doi:10.1007/s00259-012-2239-0

25. De Langen AJ, Vincent A, Velasquez LM, van Tinteren H, Boellaard R, Shankar LK, et al. Repeatability of 18F-FDG uptake measurements in tumors: a metaanalysis. J Nucl Med (2012) 53:701-8. doi:10.2967/jnumed.111.095299

Conflict of Interest Statement: The authors declare that the research was conducted in the absence of any commercial or financial relationships that could be construed as a potential conflict of interest.

Copyright (C) 2015 Mathieu, Ferrer, Carlier, Colombié, Rusu, Kraeber-Bodéré, Campion and Rousseau. This is an open-access article distributed under the terms of the Creative Commons Attribution License (CC BY). The use, distribution or reproduction in other forums is permitted, provided the original author(s) or licensor are credited and that the original publication in this journal is cited, in accordance with accepted academic practice. No use, distribution or reproduction is permitted which does not comply with these terms. 